\title{
The Design of a Suit of Frequency-Variable Controlled Equipment Based on DSP
}

\author{
SHE Yan \\ Shunde Polytechnic, Foshan 528333, China \\ Shelley186@sohu.com
}

\begin{abstract}
Keywords:DSP; frequency-variable; controlled circuit
Abstract. Due to the vital function of frequency-variable equipment in the energy-saving technology, the paper develops a suit of small power frequency -variable controlled equipment based on digital signal processor (DSP) TMS320LF2407 of American TI company. It designs holistic project of the equipment, researches and analyzes the main circuit and controlled circuit. The hardware of small DSP system, current detection module, motor speed and location module and PWM output and fault input module is introduced in detail. The experimental results indicate: As a result of TMS320LF2407 high speed and appropriative integrate-circuit, the laboratorial setting has a simpler hardware construction, more easy soft program, higher control precision and reliability in practice.
\end{abstract}

\section{Introduction}

With the development of energy-saving technology, integrated circuits are increasingly used in the field of power electronics control systems for great convenience brought to power electronics miniaturization and engineering design. The digital signal processor (DSP) applications has introduced to the drive frequency control devices, design and development of such experimental devices, digital motor control is more applicable. And as the advantage of the small size, light weight, versatility, protection function, high reliability, easily operation and so on, welcomed by metallurgy, petroleum, chemical, pharmaceutical, textile, machinery, electric power, light industry Etc. social benefits are significant. In this paper, for the requirements of the general low-power AC induction motor frequency control, developed a digital frequency control drive with TMS320LF2407 as the master chip. The whole structure is simple, with more complete functionality, can meet the inverter low-cost, high reliability, high performance requirements for general household appliances and small textiles, plastics processing and so on industrial automation production line.

\section{TMS320LF2407 DSP Overview}

The United States TI (Texas Instruments) company introduced in 1997, the TMS320LF2407 applied to industrial control and based 16-bit fixed-point TMS320C2XX digital signal processor, is the first batch members of new DSP controller. The low-cost, high-performance DSP cores and some advanced peripherals was integrated into a chip, especially suited to digital motor control applications, The DSP is a high-speed dedicated microprocessors to provide advanced, reliable and efficient signal treatment and control for high-performance transmission control. By static CMOS technology with high performance, TMS320LF2407 make the supply voltage down to 3.3V, Contain CPU with operating rate of 30MIPS (million instructions per second); The DSP integrated perfect memory and peripherals: up to $1.5 \mathrm{~K}$ word-chip data/program RAM, up to $32 \mathrm{~K}$ words flash program memory, 544 word dual-port RAM and $2 \mathrm{~K}$ words single-port RAM, expandable external memory total 192K word space; based on phase-locked loop (PLL) clock module, watch dog timer (WDT), asynchronous serial communication interface (SCI), synchronous serial peripheral interface (SPI), controller area network (CAN) ; dual 10bit ADC with 16 analog signals, one A/D converter the minimum time 500ns; 5 external interrupt; 40 individually programmed or re-used general-purpose $\mathrm{I} / \mathrm{O}$ pins; for pulse width modulation (PWM) 
control of the two event manager, each contains two general-purpose timers, 3 compare and capture unit, 2 encoding unit with the optical encoder interface, 8 PWM outputs, the PWM waveform production unit contains programmable dead time control, export the symmetric and asymmetric PWM or space vector PWM waveforms; The DSP use simulation technology based on JTAG (an international standard test protocol) scan.

As TMS320LF2407 simulation execution needs the external program memory, the system uses 16bit external program memory CY7C1021 up to 12 ns speed. With reset circuit, crystal oscillator-based PLL clock input and DSP JTAG emulation interface, compose the small system circuit.

controller area network (CAN); dual 10-bit ADC with 16 analog signals, one A/D converter the minimum time 500ns; 5 external interrupt; 40 individually programmed or re-used general-purpose I/O pins; for pulse width modulation (PWM) control of the two event manager, each contains two generalpurpose timers, 3 compare and capture unit, 2 encoding unit with the optical encoder interface, 8 PWM outputs, the PWM waveform production unit contains programmable dead time control, export the symmetric and asymmetric PWM or space vector PWM waveforms; The DSP use simulation technology based on JTAG (an international standard test protocol) scan.

As TMS320LF2407 simulation execution needs the external program memory, the system uses 16bit external program memory CY7C1021 up to 12 ns speed. With reset circuit, crystal oscillator-based PLL clock input and DSP JTAG emulation interface, compose the small system circuit[1].

\section{SVPWM Algorithm Implementation[2]}

SVPWM control technology from the perspective of the motor, focusing on the constant amplitude of the motor to obtain a circular magnetic field, the sinusoidal magnetic flux. It powered three phase sine wave voltage of the ideal circular flux trajectory as the base, with three-phase inverter have different switch modes to approximate the actual flux reference flux circle, making the track by the voltage flux space vector sum are to achieve high control performance. The purpose of three-phase inverter bridge circuit according to certain rules to control the three pairs of leg off the transistor, the DC voltage into a three-phase sinusoidal voltage output. Therefore, the three-phase bridge main circuit off state of the bridge has eight arms. Definition of the 8 switch 8 a specific combination of the basic space vector, were labeled as V4 (100), V6 (110), V2 (010), V3 (011), V1 (001), V5 (101)( The vector position fixed, the difference of 60 degree, and the vector size determined by the DC power supply voltage VD can not be controlled.) and 2 zero vector V0 (000), V7 (111) shown in Figure 1. but no direct access to any phase angle $\theta$ and the absolute value of the vector control V. 8 voltage vector using the linear combination can get more with a particular phase of the different basic space vector reference vector, according to the corresponding state to calculate the time, the control switch on and off.

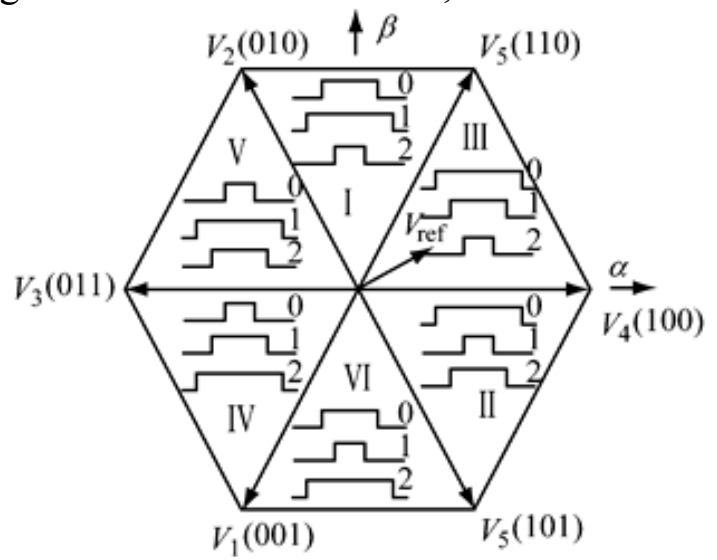

Fig. 1 SVPWM vector, sector and waveform 


\section{MAIN Circuit Design}

The main circuit of three-phase inverter use IGBT intelligent power module IPM. IPM is one kind hybrid circuit device composed of several high speed, low power IGBT and gate drive circuit integration as one. Through the use of advanced current detection type IGBT and matched with the gating circuit, it realize the efficient self protection function. Using Intelligent power module can shorten the design time and improve the reliability of the system, and make the hardware circuit simple and compact, reduce the system size. After using IPM, as driving power capacity decreasing, the device saving and comprehensive performance increasing, IPM is superior to IGBT on many occasions, have good economy.

\section{Design and Implementation of Control Circuit [3][4]}

The entire system LCI inverter main circuit, the rectifier and inverter DC link between the three main components. Control circuit design for the entire frequency control system plays a vital role in energy conservation, and micro-controller and system design is the key. Figure 2 Block diagram of the control board. LF2407 is the core of the system, it will measure the board sent the current signal, speed signal processing after the A/D conversion, and thus control the waveform of SVPWM inverter output voltage and frequency. Small systems, including DSP control board circuit, A/D sampling circuit, QEP speed detection circuit, SVPWM output, RS485/RS232 communications interface circuits, fault detection circuit, LCD display interface circuit, the switch input and output circuits, and so on .

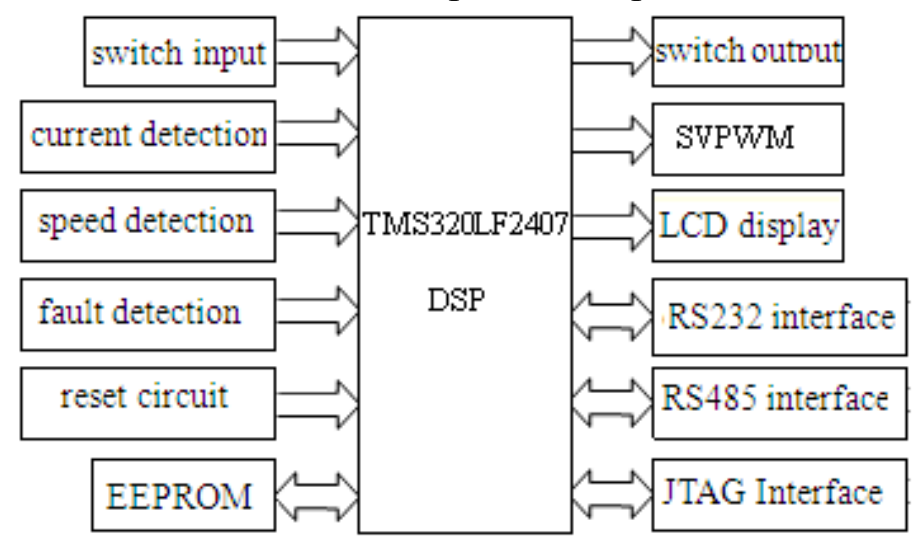

Fig.2 main control board structure

\section{Detection Circuit Design and Implementation}

Detection circuit is to detect all kinds signals, converted into the digital signal, which can be identified by DSP. Detecting circuit consists of a current detection module and the motor speed and position detection module.

These test signals are sent to directly to the TMS320LF2407 internal interface through the peripheral interface circuit, then according to the corresponding set, read the detected specific numerical[5].

\section{PWM Output and Fault Input Circuit}

Through the DSP programming, produces 6-channel programmable dead and variable output polarity of the PWM waveform, drive IPM inverter module power device of the main circuit, through a drive circuit and a photoelectric isolation circuit, obtain sine waveform of a variable-voltage and variablefrequency effect, supply electrical power[5]. 
PWM signal output request high speed isolation, otherwise it will cause the serious consequences of a short circuit. The fault input signal must be sent to the main control board after the isolation.

\section{Serial Communication Interface Circuit}

As the need of the control system with PC communication, thus require communication circuit, TMS320LF2407 itself as a SCI interface, so you can easily achieve communicates with PC. Here the communication have two functions: one is to device parameter settings through the computer to download; two is that the device send the data monitored to a computer for further processing. The system design two kinds of commonly used serial communication interface standard RS232 and RS485 interface circuit, RS232 for local data transmission; RS485 for remote data transmission.

\section{The Power Module Circuit}

TMS320LF2407 circuit board has an external 5V power supply. As the TMS320LF2407 chip power supply voltage is $3.3 \mathrm{~V}$, so in the design application board, need converse $5 \mathrm{~V}$ power to $3.3 \mathrm{~V}$ for $\mathrm{CPU}$ power supply, use TPS76833QPWP ( a low drop voltage regulator) element as an $5 \mathrm{~V} / 3.3 \mathrm{~V}$ conversion chip.

\section{Conclusion}

According to the design of frequency conversion control experimental device, by setting the motor parameters and DSP programming, the test shows that, the driving device has good performance, in line with the design requirements. By using high speed DSP chip TMS320LF2407 for the core components, constitute the frequency conversion control device, which can improve the response speed of the system. The device has the advantages of simple structure, low cost, high reliability, high performance-price ratio and good dynamic-static performance, is the best choice to drive small power motor and the automatic production line.

\section{References}

[1]LIU He-ping;YAN Li-ping; Zhang Xuefeng TMS320LF240xDSP structure principle and application [M]. Beijing: Beihang University press, 2006.11. (in Chinese)

[2]XU Ke-jun;TAO Wei-qing. DSP and its electrical and automation engineering application [M]. Beijing: Beihang University press, 2010.9. (in Chinese)

[3] SHE Yan. Realization of Frequency-Variable Speed-Adjustable System Based on AC Motor Vector Control[D]. Wuhan: Wuhan University, 2005.6. (in Chinese)

[4]WANG Shu-hong;LU Yong-jie. Based on intelligent power module in the variable frequency speed control device [J]. Journal of control theory and applications, 2007, 34 (9) : 9-13. (in Chinese)

[5]SHE Yan; SHE Yuan-hua. Fully digital vector control variable frequency speed regulating system of SVPWM [J]. Electric power automation equipment, 2006, 26 (2): 76-79. (in Chinese) 